\title{
Lesson of the month 1: Beware the atypical presentation: eosinophilic granulomatosis with polyangiitis presenting as acute coronary syndrome
}

\author{
Authors: Juergen Schiefermueller, ${ }^{A}$ Bashir Alaour, ${ }^{B}$ Alison Calver $^{C}$ and Nick Curzen ${ }^{D}$
}

\begin{abstract}
We describe the case of a 45-year-old woman presenting with troponin positive cardiac-sounding chest pain. An initial emergency angiogram demonstrated two vessel coronary disease, including a distal right coronary artery occlusion. No percutaneous coronary intervention was performed and the patient was treated medically. At re-presentation with further pain a few days later, coronary angiography demonstrated no significant coronary lesions. After consideration of other multisystem symptoms and raised eosinophil count, the patient was diagnosed with eosinophilic granulomatosis with polyangiitis (formerly known as Churg-Strauss syndrome) presenting with coronary arteritis. This case should remind physicians to be vigilant and to consider non-atherosclerotic causes of acute coronary syndrome presentation, which should not always result in a stent.
\end{abstract}

KEYWORDS: Churg-Strauss syndrome, EGPA, eosinophilic granulomatosis with polyangiitis, vasculitis

\section{Case history}

A 45-year-old woman presented to the emergency department with chest pain. Her past medical history included possible Crohn's disease, iron deficiency anaemia, chronic sinusitis, chronic back pain, chronic cough, fibromyalgia, gastro-oesophageal reflux and vitamin D deficiency. Physical examination and electrocardiogram (ECG) were unremarkable although troponin levels were elevated at $633 \mathrm{ng} / \mathrm{mL}$ (normal levels $<40 \mathrm{ng} / \mathrm{mL}$ ). Diagnostic coronary angiography showed occlusion of the distal third of the posterior descending artery branch of the right coronary artery (Fig 1A). The left anterior descending artery had a severe lesion in its mid-course and the circumflex artery was normal (Fig 1B). In view of the distal position of the posterior descending artery occlusion, the small size of the vessel and

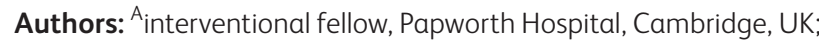
${ }^{B}$ specialist registrar in cardiology, University Hospital Southampton, Southampton, UK; ' Consultant cardiologist, University Hospital Southampton, Southampton, UK; D professor of interventional cardiology, University Hospital Southampton, Southampton, UK the duration of the occlusion ( $>48$ hours), no intervention was undertaken and the patient was treated medically. Outpatient cardiac stress magnetic resonance imaging (MRI) was arranged. She was readmitted with similar left-sided chest pain 5 days later. Serial 12-lead ECGs were non-diagnostic and troponin levels were further elevated at $1,762 \mathrm{ng} / \mathrm{mL}$. Repeat coronary angiography was undertaken; this demonstrated that the right coronary artery was now completely unobstructed (Fig 2A) and the left anterior descending artery showed minor luminal irregularities only at the site of the previously noted lesion (Fig 2B).

On further review of the case, a markedly elevated eosinophil count of $9 \times 10^{9} / \mathrm{L}$ (normal count $<0.5 \times 10^{9} / \mathrm{L}$ ) was noted. The patient complained of a 2-year history of feeling unwell, muscle pains, night sweats, tiredness and a cough with creamy sputum. Computerised tomography scan of the chest revealed no parenchymal lung lesions. Pulmonary function tests indicated an obstructive pattern although it was atypical for asthma. The patient had been resident in the UK for 25 years and had not travelled overseas in this period. No tuberculosis contacts were reported. Blood results revealed an elevated erythrocyte sedimentation rate of $50 \mathrm{~mm} /$ hour, haemoglobin $121 \mathrm{~g} / \mathrm{L}$, white cell count $15 \times 10^{9} / \mathrm{L}$ and platelets $260 \times 10^{9} / \mathrm{L}$. Anti-nuclear antibodies, anti-nuclear cytoplasmatic antibodies (ANCA), anticardiolipin antibodies, and serology for HIV and Hepatitis B and C virus were negative. Immunoglobulins, levels of complement factors C3 and C4, creatinine kinase, lupus anticoagulants and serum angiotensin were all normal. Microscopy of stool samples did not reveal any ova, cysts or parasites; serology for Strongyloides, Toxocara and Schistosoma was negative, as was sputum smear and culture for acid-fast bacilli. A presumptive diagnosis of eosinophilic granulomatosis with polyangiitis (EGPA) with coronary and myocardial involvement was made.

The patient was started on intravenous methylprednisolone $500 \mathrm{mg}$ once daily for 3 days, followed by oral prednisolone at $1 \mathrm{mg} / \mathrm{kg} /$ day. This led to rapid improvement in her overall condition and reduction in her eosinophil count. Given the transient nature of the coronary lesions, it seems most likely that the vasculitic process associated with EGPA had caused myocardial injury both directly and via coronary artery-mediated ischaemia.

The patient remained well at cardiology and rheumatology outpatient follow-up at 1-year and declined a repeat cardiac MRI scan. 

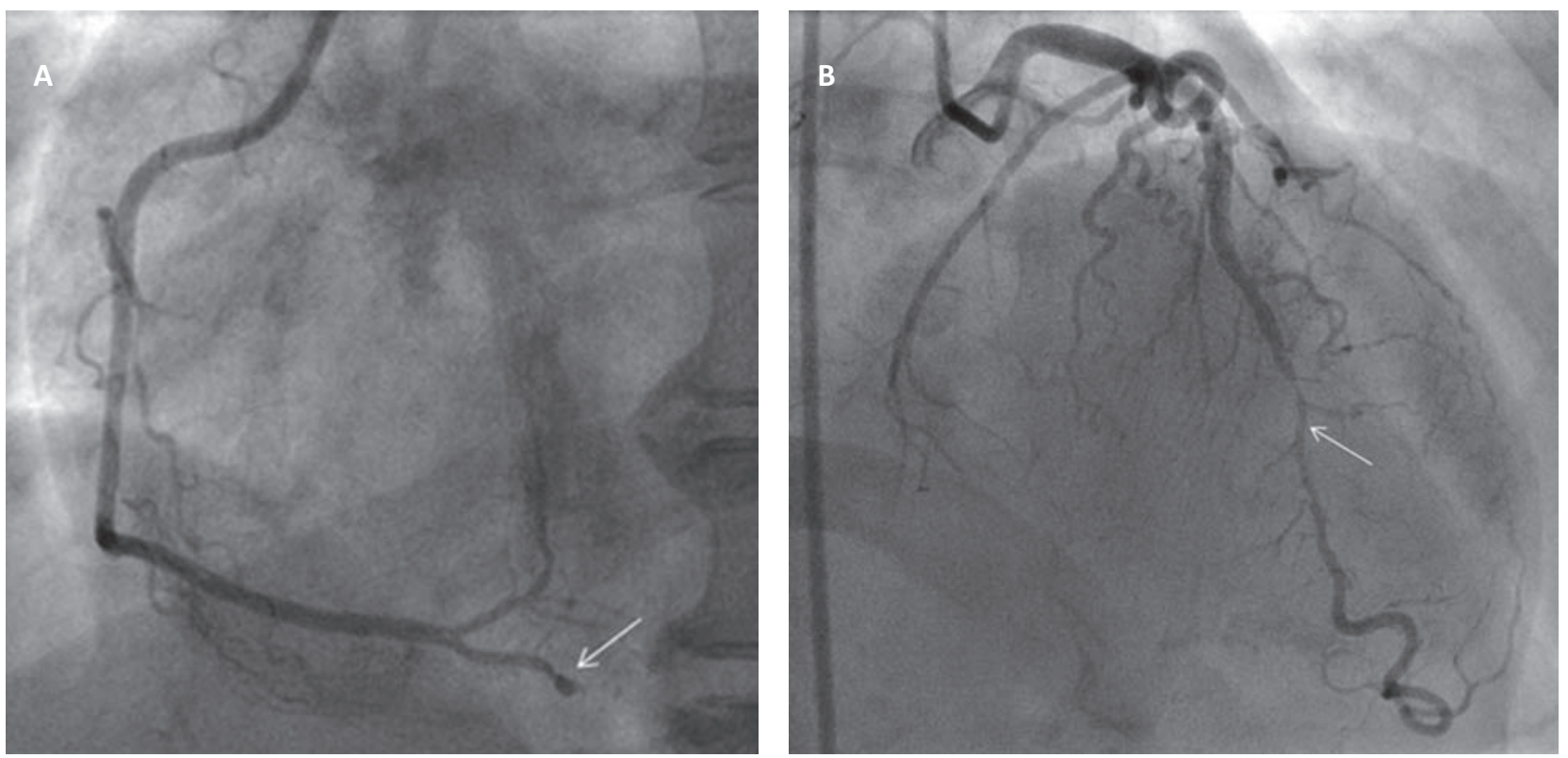

Fig 1. Initial coronary angiogram. A - left anterior oblique projection of the right coronary artery showing occlusion of the posterior descending artery branch in its mid-course (arrow); B - anterior-posterior projection with cranial angulation showing a moderate to severe appearing lesion in the mid-course of the left anterior descending artery (arrow).

\section{Discussion}

EGPA, first described in $1951^{1}$ and formerly known as ChurgStrauss syndrome, is a rare multisystem necrotising vasculitis and is characterised by the classical association of atopy, asthma, allergic rhinitis and hypereosinophilia. Histopathologically, eosinophilic infiltration and necrotising granulomas predominantly affecting the upper respiratory tract and smallmedium vessel eosinophilic giant cell vasculitis are found. Extrapulmonary manifestations can affect skin, heart, kidneys, the gastrointestinal tract and both the central and peripheral nervous system. Patients with EGPA usually present initially with
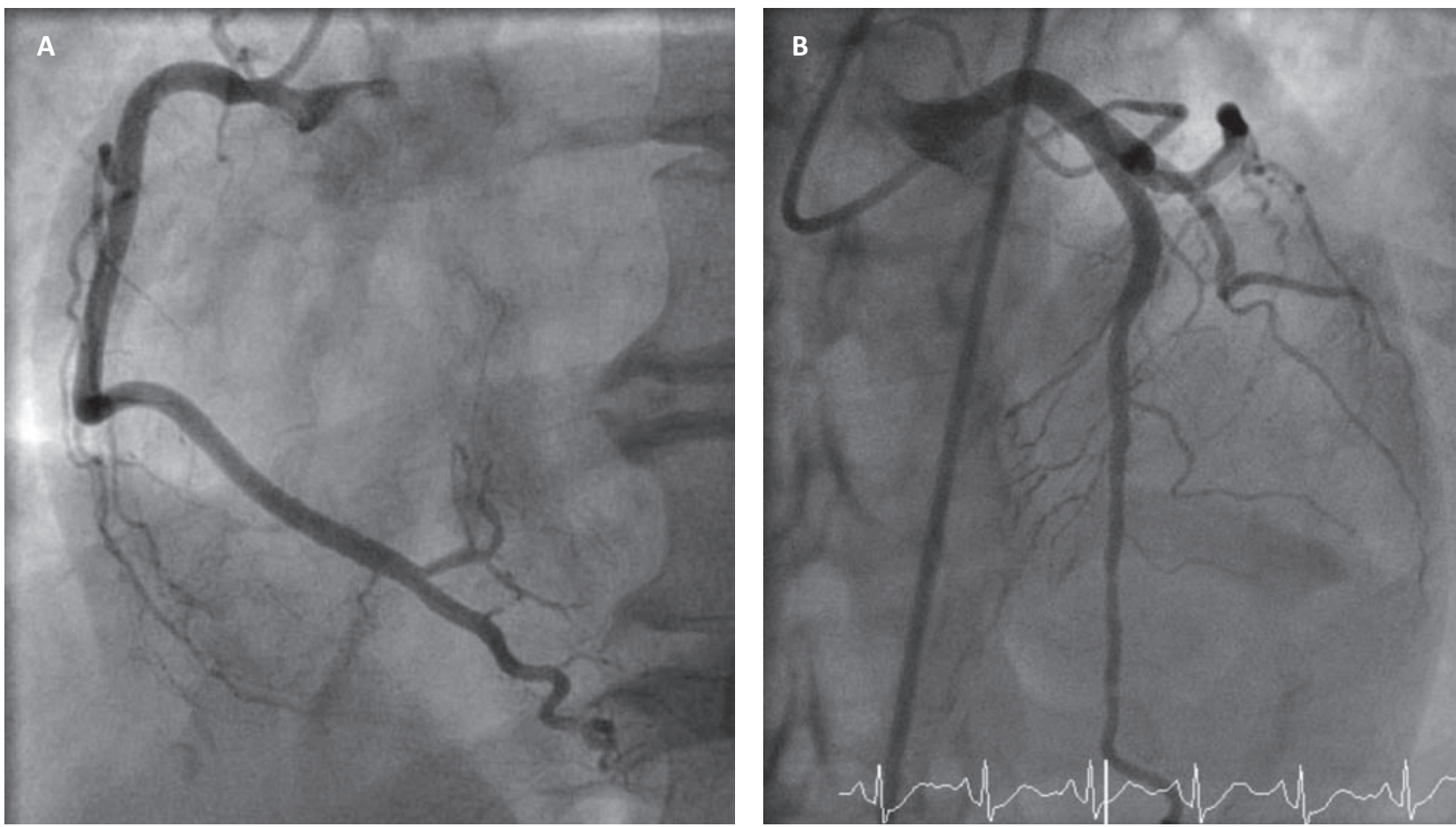

Fig 2. Repeat coronary angiogram 5 days later. A - left anterior oblique projection of the right coronary artery now showing wide patency of the posterior descending artery branch; B - left anterior oblique projection with cranial angulation now showing only minor luminal irregularities in the left anterior descending artery. 


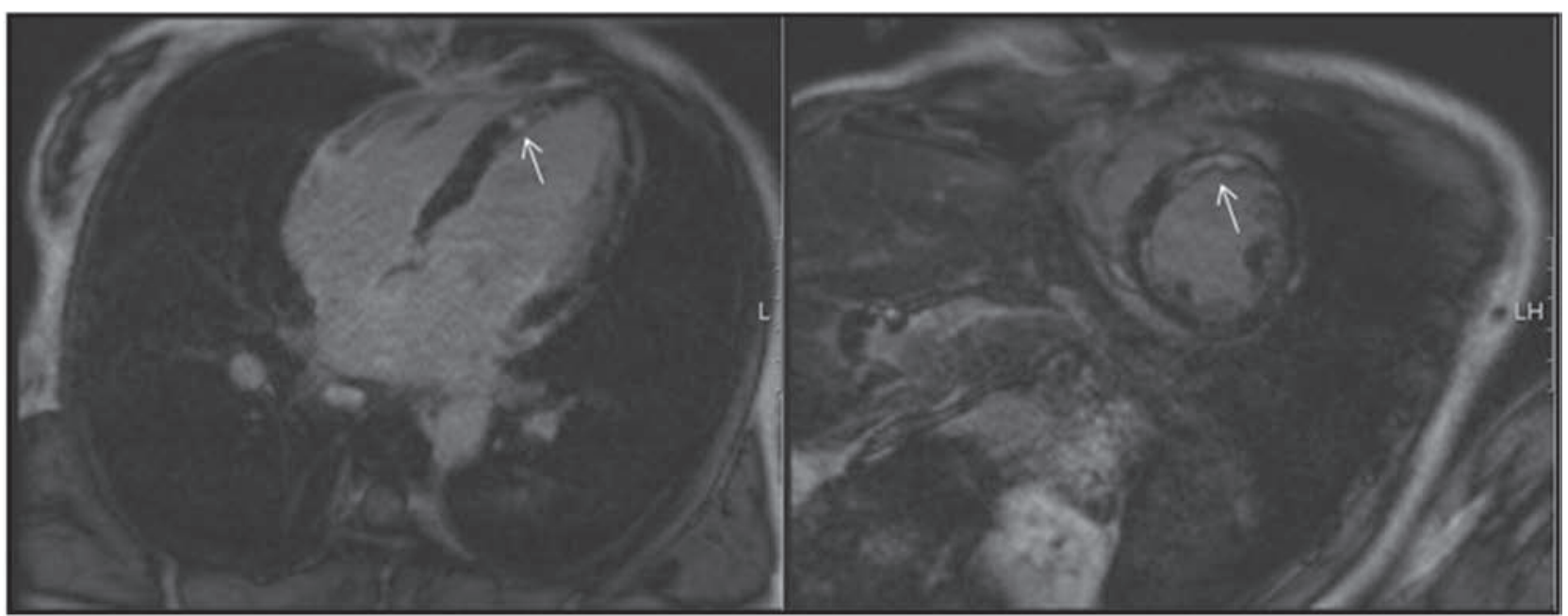

Fig 3. Cardiac magnetic resonance imaging shows patchy mid-wall late enhancement (arrows) following gadolinium contrast administration as a result of eosinophilic granulomatous myocarditis.

atopic symptoms, allergic rhinitis and asthma, with the latter often preceding the further stages by up to a decade. Fleeting pulmonary opacities, eosinophilic organ infiltrations and development of peripheral blood eosinophilia are usually the next phase before vasculitis occurs. Constitutional symptoms like malaise, fever and weight loss are common at this stage. Histological evidence of vasculitis (and/or eosinophilic infiltration and/or granulomatous inflammation) is very helpful for making a diagnosis and obtaining biopsies, usually from skin, nerve or muscle, is encouraged. ${ }^{2}$ Many classifications and diagnostic criteria for vasculitis have been developed over the years. ${ }^{3,4}$

Cardiac involvement is a serious manifestation of EGPA and is linked to about $50 \%$ of deaths. It appears to be more common in patients who are ANCA negative. Heart failure due to cardiomyopathy, pericarditis and arrhythmias is common. Regional wall motion abnormalities are the most common finding on transthoracic echocardiography in patients with EGPA, followed by mural and valvular thrombi, valvular abnormalities or pericardial effusions. Abnormalities on baseline 12-lead ECGs are frequent. Cardiac MRI with gadolinium enhancement can reveal inflammation and fibrosis.

Initial treatment of EGPA is with systemic glucocorticoids, either orally or intravenously, depending on disease severity and response to treatment. In severe cases with multi-organ involvement, cyclophosphamide for remission induction is usually added. ${ }^{5}$ Remission-maintenance therapy is with steroids, azathioprine, methotrexate and other agents. ${ }^{6}$

Epicardial coronary artery involvement in systemic vasculitis has been reported at varying frequencies, depending on the specific disease and the patient group studied. It may manifest as stenosis, thrombosis, aneurysms or dissection. ${ }^{7,8}$ In practice, patients with Kawasaki disease present with the highest incidence of coronary arteritis, with up to $20 \%$ developing coronary aneurysms.

\section{Lesson}

We present a case of a young female patient with EGPA admitted with transient coronary artery narrowing and occlusion presenting as acute coronary syndrome. The physician/cardiologist has to be vigilant to consider non- atherosclerotic, multisystem causes of acute coronary syndrome cases with atypical features.

\section{Conflicts of interest}

The authors have no conflicts of interest to declare.

\section{Author contributions}

JS wrote the first draft; $\mathrm{BA}, \mathrm{AC}$ and NC provided important contributions.

\section{Acknowledgements}

Written informed consent was obtained from the patient to publish the clinical details and images in this article.

\section{References}

1 Churg J, Strauss L. Allergic granulomatosis, allergic angiitis, and periarteritis nodosa. Am J Pathol 1951;27:277-301.

2 Groh M, Pagnoux C, Baldini C et al. Eosinophilic granulomatosis with polyangiitis (Churg-Strauss) (EGPA) Consensus Task Force recommendations for evaluation and management. Eur J Intern Med 2015;26:545-53.

3 Masi A, Hunder G, Lie J et al. The American College of Rheumatology 1990 criteria for the classification of Churg-Strauss syndrome (allergic granulomatosis and angiitis). Arthritis Rheum 1990;33:1094-100.

4 Jennette JC, Falk RJ, Bacon PA et al. 2012 Revised international Chapel Hill Consensus Conference nomenclature of vasculitides. Arthritis Rheum 2013;65:1-11.

5 Kallenberg CG. Key advances in the clinical approach to ANCAassociated vasculitis. Nat Rev Rheumatol 2014;10:484-93.

6 Bosch X, Guilabert A, Espinosa G, Mirapeix E. Treatment of antineutrophil cytoplasmic antibody associated vasculitis: a systematic review. JAMA 2007;298:655-69.

7 Pagnoux C, Guillevin L. Cardiac involvement in small and mediumsized vessel vasculitides. Lupus 2005;14:718-22.

8 Dennert RM, van Paassen P, Schalla $S$ et al. Cardiac involvement in Churg-Strauss syndrome. Arthritis Rheum 2010;62:627-34.

Address for correspondence: Dr Juergen Schiefermueller, Department of Cardiology, Papworth Hospital, Papworth Everard, Cambridge CB23 3RE, UK.

Email: juergen@schiefermueller.com 